\title{
Percepción de los estudiantes sobre las competencias sociales y personales adquiridas en el Máster en Educación Secundaria Minerva Ojeda-Delgado*, Margarita Pino-Juste y Jorge Soto-Carballo
}

\author{
Universidad de Vigo
}

Resumen: Uno de los ejes de calidad en la capacitación de los estudiantes del Máster Universitario en Formación del Profesorado de Educación Secundaria Obligatoria y Bachillerato, Formación Profesional y Enseñanzas de Idiomas, es la inserción laboral o profesional. Esto ha generado que las competencias que se ofrecen en la formación del profesorado sean más diversas y más adecuadas a la constante transformación del perfil profesional docente. Con esta investigación se quiere conocer, a través de la aplicación de un instrumento diseñado por Tribó (2008), y que pretende evaluar la percepción sobre las competencias profesionales específicas que debe obtener un profesor de secundaria, la percepción sobre el dominio de las competencias sociales y personales de 155 egresados del Máster en Educación Secundaria de la Universidad de Vigo, ya que ésta influye directamente en la enseñanza superior de calidad y en la inserción social profesional de los egresados. Los resultados no muestran diferencias significativas en función del ámbito académico con respecto a las competencias sociales. Sí encontramos diferencias con respecto a las competencias personales. Podemos concluir que es necesario plantearse si en la formación inicial del profesorado de secundaria, se responde a las competencias que necesitan para su inserción laboral.

Palabras clave: Competencias del Docente, Formación de Docentes, Evaluación de la Educación.

\begin{abstract}
Students' perception of the social and personal competencies acquired in the master's degree in secondary education

Abstract: One of the quality axes in the training of the students of the Master's degree of Secondary Education, is the labor or professional insertion. This has generated that the competences that are offered in the teacher training are more diverse and more adapted to the constant transformation of the teaching professional profile. This research is about to know, across the application of an instrument designed by Tribó (2008), and who tries to evaluate the perception on the specific professional competitions that a teacher of secundaría must obtain, the perception of the domain of social competences and personnel of 155 graduates of the Master's Degree in Secondary Education from the University of Vigo, as this directly influences quality higher education and the social-professional integration of graduates. Obtaining results that, although they do not mark significant differences according to the academic ambience with regard to the social competitions, if that they are with regard to the personal competitions. Concluding, between other things, that it is necessary to appear if really in the initial formation of the professorship of secondary, across the master's degree of secondary, it is answered to the competitions that they need for its labor insertion.
\end{abstract}

Keywords: Teacher Qualification, Teacher Education, Educational Evaluation.

Una de las funciones de la universidad es la capacitación profesional de los titulados. Así, la inserción socio-profesional del estudiante universitario se ha convertido en uno de los

Recibido: 5/12/2017 - Aceptado: 1/4/2018 - Avance online: 10/4/2018 *Correspondencia: Minerva Ojeda Delgado.

Facultade de CC. da Educación e do Deporte - Universidade de Vigo

C. P: 36005, Pontevedra, España

E-mail:minerva_ojeda@uvigo.es

Ojeda-Delgado, M., Pino-Juste, M. y Soto-Carballo, J. (2018). Percepción de los estudiantes sobre las competencias sociales y personales adquiridas en el Máster en Educación Secundaria. Journal of Psychology and Education, 13(2), 131-141, https:/l doi.org/10.23923/rpye2018.01.164 ejes de calidad para la universidad y grado de excelencia de una institución de enseñanza superior que depende, entre otros elementos, del cumplimiento de sus metas y de que éstas se adecuen a las necesidades sociales de un determinado contexto y en un momento dado. La inserción de los titulados en el mercado de trabajo será uno de los criterios de calidad de 
las universidades (Jiménez, 2007). Han sido muchos los estudios y análisis efectuados sobre la formación inicial del profesorado de secundaria en la última década (Cochran-Smith y Fries, 2005; Fernández-Soria et al., 2016; Manso y Valle, 2013; Sarramona, 2007; Zabalza, 2003).

En el estudio de Eísman, de Luna, Moreno, Moral, Rosillo y Fernández (2011) se pueden comprobar los antecedentes del proceso de formación del profesorado de secundaria hasta la actualidad.

Informes y estudios como los publicados por Eurydice European Unit (2002a, 2002b, 2003) sobre la profesión docente en Europa ponen de manifiesto que se han incorporado nuevas demandas al perfil profesional de los docentes: multiculturalidad, cuestiones de género y de convivencia, diversidad del alumnado y nuevas tecnologías, etc. En este mismo sentido el estudio TALIS ofrece un diagnóstico acerca de los procesos de formación del profesorado de secundaria (Ministerio de Educación, 2009).

Sin embargo, en términos generales se manifiesta tanto por parte las instancias profesionales de la educación como también por los estamentos políticos, una cierta insatisfacción o crítica sobre la capacidad de las instituciones de formación para dar respuesta a las necesidades actuales de la profesión docente (Marcelo, 2012; Fernández-Soria et al., 2016).

De esta forma, conocer la percepción de dominio de competencias durante el proceso de inserción socio profesional de los egresados se convierte en un medio eficaz para lograr una enseñanza superior de calidad.

Tomando como ejemplo concreto la universidad española, para su Consejo de Universidades (2002) una de las dimensiones de evaluación institucional son los resultados académicos, que han de ser analizados a largo plazo mediante el estudio de las siguientes cuestiones:

- Empleo y demanda de graduados de esa titulación. Imagen social de la titulación.

- Proporción de titulados que tienen su primer trabajo directamente relacionado con sus estudios.

- Opiniones de los titulados respecto a su formación, a la hora de buscar o encontrar empleo.

- Opiniones de los empleadores respecto a la formación de los titulados que acceden al mundo laboral.

- Valorar la adecuación entre el perfil de formación de la titulación y las características de los empleos de los egresados.

La labor docente de los profesionales de la educación en la etapa de educación secundaria requiere de competencias definidas en razón de las funciones encomendadas en la normativa vigente, las diferentes tendencias europeas en materia de formación del profesorado por competencias o bien de las propuestas que desde las universidades se realicen. Está claro que hablar de competencias profesionales implica ir más allá de las competencias científicas, didácticas o de gestión. Entendemos pues, que las competencias se deben centrar en el uso consciente de los propios conocimientos, capacidades, habilidades, destrezas, valores, actitudes y comportamientos, para resolver situaciones y problemas concretos, superando retos, cumpliendo las funciones encomendadas y alcanzando los fines propuestos. El modelo europeo sobre competencias profesionales del profesorado resalta diez competencias divididas en los ámbitos: saber, saber ser, saber hacer qué, saber hacer cómo y saber estar. Es decir, ser competente profesionalmente no sólo atañe al saber, saber qué, o el saber hacer cómo sino también ocuparse del saber ser y estar entendiendo estas desde una perspectiva holística que agrupa en la persona del educador profesional las relaciones personales y la gestión de la participación en comunidad y las habilidades relacionales, así como el conocimiento intra e interpersonal que provoca el dominio de habilidades de la gestión y promoción de valores.

El profesor Jürgen Weller (2007) destaca que existen varias dimensiones para tener en cuenta en la inserción profesional de los jóvenes que resumimos a continuación:

Primera tensión: los jóvenes tienen hoy niveles más altos de educación formal que las cohortes etáreas anteriores, pero también enfrentan 
mayores problemas de acceso al empleo, como por ejemplo en España.

Segunda tensión: los jóvenes otorgan una alta valoración al trabajo en sí, pero sus experiencias con empleos concretos suelen ser frustrantes.

Tercera tensión: Se producen fuertes contradicciones entre las expectativas de los jóvenes sobre los beneficios de la inserción en el mercado laboral y la realidad que viven en él. Las expectativas se centran en la mejoría del bienestar material individual y de la familia, así como la posibilidad de formar un hogar propio, el reconocimiento social o la posibilidad de contribuir al desarrollo de su país.

Cuarta tensión: El mercado laboral no ofrece una estabilidad mínima de empleo e ingresos y se caracteriza por una alta inestabilidad y precariedad del empleo.

Quinta tensión: el cumplimiento de las aspiraciones relacionadas con el mercado de trabajo requiere un plazo largo, en particular para alcanzar altos niveles educativos.

Sexta tensión: las mujeres jóvenes están mostrando un interés creciente por alcanzar su propia autonomía, para lo cual el empleo desempeña un papel clave, pero sin embargo siguen enfrentándose a problemas especiales de inserción laboral.

Séptima tensión: la creciente importancia de la combinación del trabajo con los estudios puede generar tensiones negativas, al afectar el rendimiento en ambos campos, o positivas, al abrir el acceso a oportunidades de otro modo negadas.

Octava tensión: los jóvenes viven la tensión entre un discurso "meritocrático" que exige grandes esfuerzos y sacrificios personales para avanzar en su educación e inserción laboral y una realidad del mercado de trabajo donde el acceso al mercado de trabajo se hace fundamentalmente a través de contactos personales y recomendaciones.

Novena tensión: el mercado exige casi siempre experiencia laboral, pero a los jóvenes le es difícil acumular experiencia y, por otra parte, el mercado laboral no reconoce la experiencia adquirida en ocupaciones a las que pueden acceder los jóvenes de bajo nivel educativo.

Décima tensión: cada vez hay mayor interés entre los jóvenes por adquirir independencia laboral y por el emprendimiento frente al escaso empleo asalariado.

Undécima tensión: los jóvenes enfrentan la tensión entre sus preferencias culturales y las pautas exigidas por un mercado de trabajo marcado por la cultura dominante.

En esta dinámica social consideramos que se debe conceder mayor importancia al entorno en el que trabaja el docente, potenciando el cambio en los contextos donde el profesorado desarrolla su labor. Se trata de pensar en el docente del siglo XXI como "expertos adaptativos", es decir, profesionales capaces de asumir un aprendizaje eficiente a lo largo de toda su vida laboral teniendo en cuenta los cambios sociales a los que estamos sometidos dentro del binomio competencia innovación (Marcelo, 2012).

En función de estas premisas, el objetivo del estudio fue describir la percepción de competencias personales y sociales de los egresados en los últimos cinco años, en el máster de educación secundaria en la Universidad de Vigo.

\section{MÉTODO}

\section{PARTICIPANTES}

La muestra estuvo conformada por 155 egresados del Máster Universitario en Formación del Profesorado de Educación Secundaria Obligatoria y Bachillerato, Formación Profesional y Enseñanzas de Idiomas, en adelante Máster de Secundaria, CEE de la Universidad de Vigo (España). El $31 \%$ son hombres y las mujeres conforman el $69 \%$. La edad media es de 30,46 siendo el mayor de 49 años y el menor de 22 y la mayoría de los egresados están solteros $(82,6 \%)$.

El reparto muestral porámbitos de conocimiento ha sido muy equilibrado. El 14,8 \% ha realizado sus estudios en grados del ámbito científico (Física, Química, ciencias del mar enfermería o biología), el 37,3 \% dentro del ámbito de las Humanidades (distintas filologías, traducción e interpretación, historia, Bellas Artes y filosofía), el 26,1 \% dentro del ámbito de las ciencias sociales y jurídicas (psicopedagogía, pedagogía, ciencias de la actividad física y deporte, magisterio, economía, 
derecho, dirección y administración de empresas) y el $21,6 \%$ en el ámbito tecnológico (arquitectura y las diferentes ingenierías).

\section{INSTRUMENTOS}

El instrumento utilizado es el diseñado y facilitado por Tribó (2008) que pretende evaluar la percepción sobre las competencias profesionales específicas que debe obtener un profesor de secundaria (véase el anexo); instrumento también utilizado por Ferrández-Berrueco, Reina \& SánchezTarazaga (2014); Se agrupan las competencias profesionales específicas en los cuatro ámbitos de competencias: científicas, metodológicas, sociales y personales. En este estudio se analizarán las dos últimas relacionadas con el saber ser y estar.

Los resultados del análisis de fiabilidad mostraron un coeficiente alfa de $\alpha=.96$ para la escala total, subescala competencias personales $\alpha=.942$, subescala competencias sociales $\alpha=.938$ lo que implica una alta fiabilidad en ambos casos (George y Mallery, 2003; Gliem y Gliem, 2003; Siitsma, 2009).

La selección de las competencias se ha realizado apoyándose, por un lado, en las aportaciones de publicaciones clásicas como el informe Delors (1996) de la UNESCO o el proyecto Tuning (2003), ya que ambos representan a colectivos importantes de expertos y de profesionales de la educación, y, por otro, adapta la propuesta de Monereo y Pozo (2007) y su enfoque coincide con las aportaciones de otros autores (Coll, 2007; De Miguel, 2005; Echevarría, 2002) tal como señala Ferrández-Berrueco y Sánchez-Tarazaga (2014).

Dentro de las competencias sociales o participativas (ámbito del saber estar) se incluyen las actitudes de colaboración en la comunidad educativa, trabajo en equipo, coordinación, acción tutorial, respeto de la normativa o investigación educativa.

Dentro de las competencias personales o interpersonales o intrapersonales (ámbito del saber ser) se incluyen las actitudes de control emocional, toma de decisiones, asunción de deberes y responsabilidades o educación en valores.

\section{PROCEDIMIENTO}

El estudio se llevó a cabo siguiendo las normas deontológicas reconocidas por la Declaración de Helsinki (revisión de Hong-Kong, septiembre de 1989) y de acuerdo con las recomendaciones de Buena Práctica Clínica de la Comunidad Económica Europea (documento 111/3976/88 de julio de 1990) y la normativa legal vigente española que regula la investigación.

El cuestionario se administró de manera colectiva a los egresados en la universidad de Vigo mediante un cuestionario on line durante el curso 2016-17; habiendo concluido sus estudios del Máster.

\section{ANÁLISIS DE DATOS}

Se llevó a cabo, en primer lugar, un análisis descriptivo de los ítems, porcentajes, media, desviación típica, así como los índices de asimetría y curtosis para evaluar el comportamiento normal de las variables.

A continuación, se emplea la prueba de Kolmogorov Smirnov con el propósito de decidir de un modo riguroso, si la muestra de la que se dispone procede o no de una distribución normal $y$, seguidamente, se realizaron pruebas T y ANOVA de comparación de medias independientes con un nivel de significación de $p<.05$. Se ha utilizado un nivel de confianza de.05 y, para el análisis de los datos se emplearon el programa estadístico SPSS 23.0

\section{RESULTADOS}

La mayoría de los egresados del Máster en secundaria no trabaja en la enseñanza (61,3\%) aunque algunos de ellos trabajan impartiendo clases particulares, pero otros muchos de camareros, comerciales o empleos relacionados con la administración.

Como podemos comprobar en los resultados de la Tabla 1 los docentes encuestados opinan en un alto porcentaje que no poseen competencias para dinamizar al alumnado con el fin de crear reglas de convivencia $(64,6 \%)$, ni para fomentar las relaciones de los centros educativos con el entorno $(63,3 \%)$, ni siquiera para participar en 
Tabla

Porcentajes de los diferentes ítems según su graduación $(N=155)$

\begin{tabular}{|c|c|c|c|c|}
\hline & 0 & 1 & 2 & 3 \\
\hline COMPETENCIAS SOCIALES (Saber estar) & \multicolumn{4}{|c|}{ Porcentajes } \\
\hline 1. Dinamizar el alumnado para construir reglas de convivencia & 24.5 & 40.1 & 27.3 & 9.2 \\
\hline 2. Potenciar en el alumnado una actitud de ciudadanía crítica & 20.6 & 38.1 & 31.0 & 10.3 \\
\hline 3. Tener una actitud colaborativa con la comunidad educativa & 17.4 & 34.8 & 32.9 & 14.8 \\
\hline 4. Trabajar en equipo con otros profesionales & 16.8 & 25.8 & 38.1 & 19.4 \\
\hline 5. Participar en los proyectos del centro (Plan Acción Tutorial, Calidad...) & 26.5 & 35.5 & 29.7 & 8.4 \\
\hline 6. Facilitar la comunicación con el alumnado y familias & 23.9 & 35.5 & 29.7 & 10.9 \\
\hline 7. Respetar y saber aplicar la normativa del sistema educativo & 16.1 & 37.4 & 37.4 & 9.0 \\
\hline 8. Fomentar la relación del centro educativo con los agentes del entorno & 25.2 & 38.1 & 29.7 & 7.1 \\
\hline 9. Participar en proyectos de investigación educativa & 31.6 & 32.3 & 24.5 & 11.6 \\
\hline COMPETENCIAS PERSONALES (Saber ser) & \multicolumn{4}{|c|}{ Porcentajes } \\
\hline 1. Tener un buen conocimiento de mí mismo y de mis capacidades & 20.0 & 24.5 & 37.4 & 18.0 \\
\hline 2. Saber tomar decisiones individualmente & 17.4 & 27.1 & 36.1 & 19.4 \\
\hline 3. Asumir responsabilidades en el centro y liderazgo, si es necesario & 29.0 & 32.3 & 25.2 & 13.6 \\
\hline 4. Fomentar un clima basado en el respeto y en la libertad de expresión & 16.1 & 21.9 & 40.0 & 21.9 \\
\hline 5. Tener autocontrol y equilibrio emocional, resistente a la frustración & 29.7 & 24.5 & 29.0 & 16.7 \\
\hline 6. Educar en valores al alumnado (y ser consciente de ello) & 11.6 & 29.0 & 31.6 & 27.0 \\
\hline 7. Asumir la necesidad de desarrollo profesional continuado & 11.0 & 23.9 & 38.1 & 27.1 \\
\hline
\end{tabular}

Leyenda: $\mathrm{O}=$ Nada importante, 1 = algo importante, 2 = importante y 3 = muy importante

\begin{tabular}{|c|c|c|c|}
\hline \multicolumn{4}{|c|}{$\begin{array}{c}\text { Tabla } 2 \\
\text { Porcentaje de egresados según nivel de competencias }(N=155)\end{array}$} \\
\hline & & Frecuencia & Porcentaje \\
\hline \multirow{4}{*}{$\begin{array}{l}\text { Competencias sociales } \\
\qquad M=1.31\end{array}$} & Bajo & 54 & 34.8 \\
\hline & Medio & 78 & 50.3 \\
\hline & Alto & 23 & 14.8 \\
\hline & Total & 155 & 100.0 \\
\hline \multirow{2}{*}{ Competencias personales } & Bajo & 47 & 30.3 \\
\hline & Medio & 67 & 43.2 \\
\hline \multirow[t]{2}{*}{$M=1.57$} & Alto & 41 & 26.5 \\
\hline & Total & 155 & 100.0 \\
\hline
\end{tabular}

los proyectos de centro (62\%). La competencia social que consideran más adquirida es la de trabajar en equipo con otros profesionales (57,5\%).

Con respecto a las competencias personales, los docentes noveles consideran que tienen un mayor dominio de estas y opinan que tienen un buen conocimiento de sí mismo y de sus capacidades $(55,4 \%)$ o saben tomar decisiones individualmente $(55,5 \%)$, saben educar en valores $(58,6 \%)$ y saben fomentar un clima basado en el respeto y en la libertad de expresión $(61,9 \%)$, o asumen que necesitan un desarrollo profesional continuo $(65,2 \%)$.

Los datos descritossoncoherentessiagrupamos los resultados. Como podemos comprobar en la Tabla 2, la mayoría del alumnado tienen una percepción de sus competencias medio $(50,3 \%)$ e incluso el $34,8 \%$ piensa que tiene bajas competencias sociales. Con respecto a las 


\begin{tabular}{|c|c|c|}
\hline \multicolumn{2}{|c|}{ Tescriptivos de las competencias social y personal $(N=155)$} \\
\hline Media & Competencia social & Competencia personal \\
\hline Mediana & 1,31 & 1,54 \\
Moda & 1,33 & 1,58 \\
Desv. típ. & 1,44 & 1,86 \\
Asimetría & 0,75 & $-0,85$ \\
Curtosis & 0,08 & $-0,79$ \\
Mínimo & $-0,50$ & 0 \\
Máximo & 0 & 3 \\
\hline
\end{tabular}

competencias personales el 43,2 \% opina que tiene unas competencias medias pero el 30,3\% piensa que las tiene bajas.

La media en competencia personal es relativamente baja, como puede observarse en la abla 3, aunque levemente superior la media en competencia social ya que ambas quedan lejos de la puntuación máxima que es 3 .

La curtosis es en ambas distribuciones negativa lo que quiere decir que la distribución está menos apuntada que la normal, por tanto platicurtica. Mientras que la asimetría es negativa, es decir los datos se agrupan ligeramente a la izquierda de la media en el caso de la competencia personal y positiva o a la derecha en el caso de la competencia social.

Como se puede apreciar en la Tabla 4, no existen diferencias en función del género ni en función del estado civil ni de si trabajan o no en la enseñanza, aunque sienten que tienen mayores

Tabla 4

Resultados de $t$ de student con respecto al género, estado civil y si trabaja o no en la enseñanza $(N=155)$

\begin{tabular}{|c|c|c|c|c|c|c|c|}
\hline \multirow[b]{2}{*}{ V.D } & \multirow[b]{2}{*}{ Variable } & \multirow[b]{2}{*}{$\mathrm{N}$} & \multirow[b]{2}{*}{ Media } & \multicolumn{2}{|c|}{ Prueba de Levene } & \multicolumn{2}{|c|}{ Prueba t de Student } \\
\hline & & & & $\mathrm{F}$ & Sig. & $t$ & $\begin{array}{c}\text { Sig. } \\
\text { (bilateral) }\end{array}$ \\
\hline Competencia social & $\begin{array}{l}\text { Hombre } \\
\text { Mujer }\end{array}$ & $\begin{array}{c}48 \\
107\end{array}$ & $\begin{array}{l}1.25 \\
1.34\end{array}$ & 0.77 & .379 & -0.720 & .473 \\
\hline Competencia personal & $\begin{array}{l}\text { Hombre } \\
\text { Mujer }\end{array}$ & $\begin{array}{c}48 \\
107\end{array}$ & $\begin{array}{l}1.38 \\
1.60\end{array}$ & 0.65 & .420 & -1.49 & .136 \\
\hline Competencia social & $\begin{array}{l}\text { Soltero } \\
\text { Casado }\end{array}$ & $\begin{array}{l}128 \\
27\end{array}$ & $\begin{array}{l}1.32 \\
1.32\end{array}$ & 1.09 & .317 & 0.020 & .984 \\
\hline Competencia personal & $\begin{array}{l}\text { Soltero } \\
\text { Casado }\end{array}$ & $\begin{array}{l}128 \\
27\end{array}$ & $\begin{array}{l}1.58 \\
1.37\end{array}$ & 0.54 & .462 & 0.239 & .214 \\
\hline Competencia social & $\begin{array}{l}\mathrm{SI} \\
\mathrm{NO}\end{array}$ & $\begin{array}{l}58 \\
95\end{array}$ & $\begin{array}{l}1.26 \\
1.35\end{array}$ & 2.02 & .158 & -0.753 & .452 \\
\hline Competencia personal & $\begin{array}{l}\mathrm{SI} \\
\mathrm{NO}\end{array}$ & $\begin{array}{l}58 \\
95\end{array}$ & $\begin{array}{l}1.51 \\
1.56\end{array}$ & 1.88 & .172 & -0.387 & .702 \\
\hline
\end{tabular}


competencias tanto personales como sociales las mujeres y los que no trabajan en docencia. Los solteros y casados consideran que tienen el mismo nivel de competencias sociales $y$, aunque la percepción de dominio de competencias personales es mayor en los solteros no existen diferencias significativas.

Con respecto a las diferencias en función del ámbito académico de la Tabla 5, no existen diferencias con respecto a las competencias sociales, pero si con respecto a las competencias personales siendo los egresados de humanidades quienes perciben un mayor dominio en este tipo de competencias.

\section{DISCUSIÓN}

En el ámbito mundial, se han logrado avances enormes cara al de objetivo de la educación primaria universal, lo que ha llevado a una mayor demanda de educación secundaria y como consecuencia, más jóvenes y adultos jóvenes están entrando en los mercados de trabajo formales e informales. Por tanto, resulta importante asegurar que las escuelas secundarias enseñen habilidades relevantes para el mundo del trabajo (Jayaram y Engmann, 2014). Sin embargo, Moreno (2006) señala que el número de profesorado no cualificados suele ser mucho más alto en la enseñanza secundaria que en la primaria, en casi todos los países en desarrollo. De ahí la importancia de que el profesorado de secundaria desarrolle competencias personales y sociales para el desempeño eficaz de su labor docente. De hecho, Jayaram y Engmann (2014) señalan que los empleadores están buscando tres tipos clave de habilidades: cognitiva, no cognitiva y técnica. Aunque las habilidades cognitivas técnicas y básicas siguen siendo importantes para la actividad laboral, las habilidades transferibles y no cognitivas como la comunicación, la resolución de problemas, la puntualidad y la flexibilidad son cada vez más importantes, particularmente para la economía informal. Normalmente estas habilidades se trasladan por imitación de modelos.

Dada la importancia, por tanto, de las habilidades sociales y personales para el desarrollo

\begin{tabular}{|c|c|c|c|c|c|c|}
\hline \multicolumn{7}{|c|}{$\begin{array}{c}\text { Tabla } 5 \\
\text { Diferencia de medias de las diferentes competencias con respecto al ámbito }(N=155)\end{array}$} \\
\hline & & $\mathrm{n}$ & Media & $\mathrm{F}$ & Sig. & Bonferroni \\
\hline \multirow{5}{*}{$\begin{array}{l}\text { Competencias } \\
\text { sociales }\end{array}$} & Ciencias & 23 & 1.43 & \multirow{5}{*}{0.581} & \multirow{5}{*}{.628} & \multirow{5}{*}{$\begin{array}{l}\text { No existen } \\
\text { diferencias }\end{array}$} \\
\hline & Humanidades & 57 & 1.32 & & & \\
\hline & $\begin{array}{l}\text { C. Sociales y } \\
\text { jurídicas }\end{array}$ & 40 & 1.35 & & & \\
\hline & Ingeniería & 35 & 1.18 & & & \\
\hline & Total & 155 & 1.32 & & & \\
\hline \multirow{5}{*}{$\begin{array}{l}\text { Competencias } \\
\text { personales }\end{array}$} & Ciencias & 23 & 1.66 & \multirow{5}{*}{2.745} & \multirow{5}{*}{.45} & \multirow{5}{*}{$\begin{array}{l}\text { Humanidades / } \\
\text { Ingeniería }=.037\end{array}$} \\
\hline & Humanidades & 57 & 1.70 & & & \\
\hline & $\begin{array}{l}\text { C. Sociales y } \\
\text { jurídicas }\end{array}$ & 40 & 1.54 & & & \\
\hline & Ingeniería & 35 & 1.19 & & & \\
\hline & Total & 155 & 1.55 & & & \\
\hline
\end{tabular}


profesional del docente, es importante determinar el nivel de adquisición de estas. En nuestro estudio queda patente que el profesorado de secundaria egresados en los últimos cinco años consideran que no tienen consolidadas están competencias y creen que tienen mayor habilidades personales que sociales destacando por más de la mitad de ellos la habilidad para trabajar en equipo con otros profesionales y que pueden tomar decisiones individualmente que saben educar en valores en un clima de respeto y libertad de expresión y asumen que necesitan un desarrollo profesional continuo. También en el estudio de Eísman, de Luna, Moreno, Moral, Rosillo y Fernández (2011) una de las competencias más valoradas por los estudiantes era fomentar el espíritu crítico, reflexivo y emprendedor o fomentar el respeto a los Derechos Humanos y a los principios de accesibilidad universal, igualdad, no discriminación y los valores democráticos y de la cultura de la paz.

De hecho, solamente un $14,8 \%$ considera que tiene altas competencias sociales y el $26,5 \%$ altas competencias personales. En el estudio de Ferrández-Berrueco y Sánchez-Tarazaga (2014) también el grupo de competencias mejor puntuado son las competencias personales ligadas a los rasgos psicológicos del docente.

Estas opiniones se mantienen en ambos sexos, en función del estado civil, o de si trabajan o no en la enseñanza y solamente aparecen diferencias con respecto al ámbito académico, siendo los egresados de humanidades quienes perciben un mayor dominio de competencias personales.

Estos resultados llevan a preguntarnos si realmente en la formación inicial del profesorado de secundaria, a través del máster de secundaria, se responde a las competencias que necesitan para su inserción laboral (Moll, 2011 1). De hecho, Moreno Olmedilla (2006) señala que existe un desajuste entre las nuevas competencias clave que se exigen a los graduados de educación secundaria en la sociedad del conocimiento, y las competencias docentes con las que se les forman en las diferentes universidades.

Desde el año 2007 se ha seguido debatiendo sobre el dominio de estas competencias. En este año se establecieron los principios europeos comunes para las competencias y cualificaciones del profesorado enmarcados en el Programa de Educación y Formación para el 2010 y se considera que la formación inicial del profesorado, y a lo largo de toda su carrera profesional, son la clave para el desarrollo de una economía competitiva y dinámica (Real Decreto 1393/2007)

Vilches y Gil (2010) recuerdan la necesidad del máster de secundaria de responder a los serios problemas detectados en la educación española. Para ello, según los autores, resulta imprescindible, y estamos en total sintonía con esta idea, obtener una coherencia entre la orientación pedagógica de las diferentes materias del máster y las estrategias de enseñanza que se pretende ejerciten el futuro profesorado con su alumnado. De ahí, la importancia de seleccionar adecuadamente al profesorado que imparte esta formación en el máster. Además, se señala también la importancia del prácticum ya que es preciso hacer el máximo esfuerzo para "implicar como tutores a aquel profesorado que son capaces de generar el interés de los estudiantes, de lograr su aprendizaje y resolver situaciones de conflicto" (Vilches y Gil, 2010:663).

\section{- Conflicto de intereses}

Los autores declaran no tener ningún conflicto de intereses.

\section{REFERENCIAS}

Cochran-Smith, M., \& Fries, M. (2005). The AERA Panel on Research and Teacher Education: Context and goals. In M. CochranSmith \& K. Zeichner (Eds.), Studying teacher education: The report of the AERA Panel on Research and Teacher Education (pp. 37-68). Mahwah, NJ: Erlbaum. Cochran-Smith, M., \& Zeichner, K.

Coll, C. (2007). Una encrucijada para la educación escolar. Cuadernos de Pedagogía, 370, 19-23.

Consejo de Universidades (2002). Plan Nacional de Evaluación de la Calidad de las Universidades. Informe anual de la Segunda Convocatoria. Madrid. Ministerio de Educación y Ciencia.

Delors, J. (1996): Informe a la Unesco de la Comisión Internacional sobre la Educación para el Siglo XXI. Madrid: Santillana. 
De Miguel, M. (2005). Metodologías de enseñanza para el desarrollo de competencias. Orientaciones para el profesorado universitario ante el espacio Europeo de Educación Superior. Madrid: Alianza.

Echeverría, B. (2002). Gestión de la Competencia de Acción Profesional. Revista de Investigación Educativa, 20(1), 7- 43.

Eísman, L. B., de Luna, E. B., Moreno, E. M. O., Moral, M. P., Rosillo, M. A. R., y Fernández, M. T. (201 1). Valoración por parte del alumnado de las competencias que se pretenden conseguir con el Máster Universitario de profesorado de educación secundaria obligatoria, bachillerato, formación profesional y enseñanza de idiomas. Bordón. Revista de Pedagogía, 63(3), 57-74.

Eurydice European Unit (2002a). La profesión docente en Europa: Perfil, tendencias y problemática. Informe II. Oferta y demanda. Educación secundaria inferior general. Bruselas: Eurydice.

Eurydice European Unit (2002b). Las Competencias Clave: Un concepto en expansión dentro de la educación general obligatoria. Bruselas: Eurydice.

Eurydice European Unit (2003). La profesión docente en Europa: Perfil, tendencias e intereses. Informe I. Formación inicial y transición a la vida laboral. Educación Secundaria inferior general. Bruselas: Eurydice.

Fernández-Soria, J. M., López, M., Cruz, J. I., Bascuñán, J., Mengual, S., García, S., Lloret, C., y Grau, R. (2016)

Ferrández-Berrueco, R. y Sánchez-Tarazaga, L. (2014). Competencias docentes en secundaria. Análisis de perfiles de profesorado. Relieve, 20 (1), 1-20. Doi: 10.7203/relieve.20.1.3786.

George, D., y Mallery, P. (2003). SPSS for Windows step by step: A simple guide and reference. 11.0 update ( $4^{a}$ ed.). Boston, MA: Allyn \& Bacon.

Gliem, J., y Gliem, R. (2003). Calculating, Interpreting, and Reporting Cronbach's Alpha Reliability Coefficient for Likert-Type Scales. Conference in Adult, Continuing and Community Education. Midwest Research to Practice. The Ohio State University, Columbus. Jayaram, S. y Engmann, M. (2014). Developing skills for employability at the secondary level: Effective models for Asia. Prospects, 44: 221233. Doi: $10.1007 / \mathrm{s} 111125-014-9302-5$

Jiménez A. (2007). Orientación profesional en los titulados universitarios. La profesionalización como criterio de calidad. Salamanca, Agencia Española de Cooperación Internacional.

Manso, J. y Valle, J. M. (2013). La formación inicial del profesorado de secundaria en la Unión Europea. Revista Española de Educación Comparada, 22, 165-184

Marcelo, C. (2012). Empezar con buen pie: inserción a la enseñanza para profesores principiantes. Revista Olhar de Professor.

Ministerio de Educación (2009). TALIS (OECD). Estudio internacional sobre la Enseñanza y Aprendizaje. Informe español. Madrid: Secretaría de Estado de Educación y Formación Profesional. Dirección General de Evaluación y Cooperación Territorial. Instituto de Evaluación Moll, V. F. (2011). Competencias profesionales en la formación inicial de profesores de matemáticas de secundaria. UNIÓN: Revista Iberoamericana de Educación Matemática, (26), 7-8.

Monereo, C., y Pozo J.I. (2007). Competencias para convivir en el siglo XXI. Cuadernos de Pedagogía, 370, 11-18.

Moreno, J. M. (2006). Profesorado de Secundaria y Calidad de la Educación: Un marco de opciones políticas para la formación y el desarrollo profesional docente. Profesorado. Revista de currículum y formación del profesorado, 10 (1), 1 - 21.

Sarramona, J. (2007). Las competencias profesionales del profesorado de secundaria. ESE. Estudios sobre educación. 12, 31-40.

Sijtsma, K. (2009). On the use, the misuse and the very limited of the Cronbach's alpha. Psychometrika, 74(1) 107-120. https://doi. org/10.1007/S1 1336-008-9101-0

Tribó, G. (2008). El nuevo perfil profesional de los profesores de secundaria, Educación XX1 (11), 183-209.

Proyect Tuning (2003): Tuning Educational Structures in Europe. Informe final. Proyecto piloto. Fase 1. Bilbao: Universidad de Deusto. Vilches, A., y Gil, D. (2010). Máster de formación 
inicial del profesorado de enseñanza secundaria. Algunos análisis y propuestas.

Revista Eureka sobre Enseñanza y Divulgación de las Ciencias, 7(3), 661-666.

Weller, J. (2007). La inserción laboral de los jóvenes: características, tensiones y desafíos. Revista de la CEPAL, 92, 61-82.

Zabalza, M. A. (2003). Competencias docentes del profesorado universitario: calidad y desarrollo profesional. Madrid: Narcea.

\section{ANEXO \\ CUESTIONARIO SOBRE COMPETENCIAS DOCENTES \\ (TRIBÓ, 2008)}

\section{HOJA DE DATOS PERSONALES}

Edad:

Sexo:

$\square \mathrm{H}$

$\square M$

Estado civil:

$\square$ Soltera/o

$\square$ Casada/o

$\square$ Viuda/o

$\square$ Separada/o

Licenciado / Graduado en:

Áreas de conocimiento:

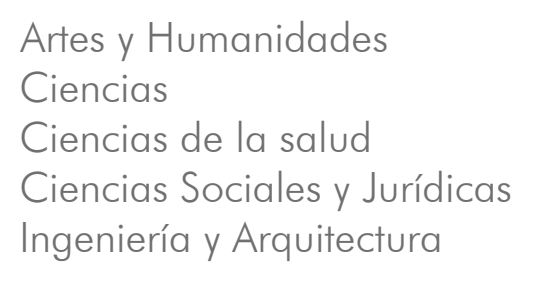

Año de graduación:

La Formación en educación la he realizado:

$\square$ Máster

$\square$ CAP

En la actualidad está trabajando en la enseñanza:

$\square$ SI

$\square \mathrm{NO}$

En qué curso académico comenzó a trabajar en la enseñanza:

Trabaja en otra actividad fuera de la enseñanza:

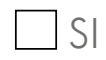

Cuál:

A continuación, se presentan un listado de competencias, donde deberá indicar el grado de importancia que tiene para usted cada una de ellas (siendo $0=$ nada importante, $1=$ algo importante, $2=$ importante y $3=$ muy importante). 


\section{COMPETENCIAS CIENTíFICAS (Saber)}

1.1. Conocer los contenidos de la materia que imparto

1.2. Estar actualizado sobre las novedades en la materia

1.3. Conocer la historia y evolución de la materia de la que soy profesor

1.4. Saber transferir mis conocimientos para que el alumno aprenda

1.5. Saber planificar y organizar los contenidos

1.6. Tener formación en pedagogía y psicología aplicada a jóvenes

1.7. Conocer estrategias educativas innovadoras

1.8. Conocer las TIC (nuevas tecnologías, Internet)

1.9. Tener un alto nivel de competencia lingüística en lenguas oficiales

1.10. Conocer una lengua extranjera para poder dar clases (inglés)

2. COMPETENCIAS METODOLÓGICAS (Saber hacer)

2.1. Gestionar el clima del aula (ambiente de trabajo, confianza, diálogo...)

2.2. Aplicar estrategias para que los alumnos trabajen en grupo

2.3. Fomentar dinámicas para la cohesión de la clase

2.4. Dar respuesta a los problemas de diversidad en el aula

2.5. Elaborar propuestas para alumnos con necesidades educativas

2.6. Saber resolver conflictos dentro del aula

2.7. Desarrollar las tutorías y saber orientar académica y profesionalmente

2.8. Introducir elementos de mejora, tras una reflexión sobre mi práctica

2.9. Dominar las competencias comunicativas verbales y no verbales

2.10. Utilizar diferentes técnicas y sistemas de evaluación del alumnado

2.11. Explorar conocimientos previos del alumno mediante evaluación inicial

2.12. Realizar un seguimiento del grado de aprendizaje de los alumnos

2.13. Utilizar la evaluación como herramienta de mejora de aprendizaje

2.14. Diseñar una programación didáctica

2.15. Saber elaborar materiales didácticos

2.16. Dominar las TIC para utilizarlas como recurso en clase

2.17. Educar al alumnado para la información y comunicación tecnológica

\section{COMPETENCIAS SOCIALES (Saber estar)}

3.1. Dinamizar el alumnado para construir reglas de convivencia

3.2. Potenciar en el alumnado una actitud de ciudadanía crítica

3.3. Tener una actitud colaborativa con la comunidad educativa

3.4. Trabajar en equipo con otros profesionales

3.5. Participar en los proyectos del centro (Plan Acción Tutorial, Calidad...)

3.6. Facilitar la comunicación con alumnos y familias

3.7. Respetar y saber aplicar la normativa del sistema educativo

3.8. Fomentar la relación del centro educativo con los agentes del entorno

3.9. Participar en proyectos de investigación educativa

\section{COMPETENCIAS PERSONALES (Saber ser)}

4.1. Tener un buen conocimiento de mí mismo y de mis capacidades

4.2. Saber tomar decisiones individualmente

4.3. Asumir responsabilidades en el centro y liderazgo, si es necesario

4.4. Fomentar un clima basado en el respeto y en la libertad de expresión

4.5. Tener autocontrol y equilibrio emocional, resistente a la frustración

4.6. Educar en valores a los alumnos (y ser consciente de ello)

4.7. Asumir la necesidad de desarrollo profesional continuado

\begin{tabular}{|c|c|c|c|}
\hline 0 & 1 & 2 & 3 \\
\hline & & & \\
\hline & & & \\
\hline & & & \\
\hline & & & \\
\hline & & & \\
\hline & & & \\
\hline & & & \\
\hline & & & \\
\hline & & & \\
\hline & & & \\
\hline & & & \\
\hline & & & \\
\hline & & & \\
\hline & & & \\
\hline & & & \\
\hline & & & \\
\hline & & & \\
\hline & & & \\
\hline & & & \\
\hline & & & \\
\hline & & & \\
\hline & & & \\
\hline & & & \\
\hline & & & \\
\hline & & & \\
\hline & & & \\
\hline & & & \\
\hline & & & \\
\hline & & & \\
\hline & & & \\
\hline & & & \\
\hline & & & \\
\hline & & & \\
\hline & & & \\
\hline & & & \\
\hline & & & \\
\hline & & & \\
\hline & & & \\
\hline & & & \\
\hline & & & \\
\hline & & & \\
\hline & & & \\
\hline & & & \\
\hline & & & \\
\hline & & & \\
\hline & & & \\
\hline
\end{tabular}

Revista de Psicología y Educación / Journal of Psychology and Education, 2018, 13(2), 131 -141 www.rpye.es Doi: https://doi.org/10.23923/rpye2018.01. 164 